\title{
Materials science aspects of the research directions of electrochemistry
}

\author{
László Péter ${ }^{1}$ \\ Received: 20 February 2020 / Revised: 20 February 2020 / Accepted: 11 March 2020 / Published online: 19 March 2020 \\ (C) The Author(s) 2020
}

\begin{abstract}
This opinion article summarizes a few electrochemical research directions whose weight is likely to increase in the near future. Connection between materials science and electrochemistry is highly emphasized, while education is also considered as a key issue.
\end{abstract}

Electrochemistry proved to be an ever-expanding field since the discovery of the Volta column. The milestone list of the quantitative treatment of electrochemical phenomena includes the Faraday law that connects chemical quantity with charge, Tafel law that bridges (although somewhat heuristically) the current passing through an electrode and the electrode potential measured, and the description of the polarization curves as given by Erdey-Grúz and Volmer that defines the electrode potential (difference) as a driving force and the current as a consequence. The development of semiconductor-based devices ensured that, in accord with the contemplation that considers the electrode potential as the primary variable, potential-controlled experiments can be done. While the accuracy of the electronic control, the speed of the measurements and variety of methods improved significantly in the second half of the twentieth century; the digital control of the instrumentation was a significant step especially in the combination of methods and data acquisition related to electrochemical experiments. Of course, it would be an obscure idea to regard electrochemical instrumentation and methodology as being ready. However, their development is rather difficult to predict in the opinion of the author, and any new discovery will certainly be based on the coincidence of various factors, among which personal excellence is an important one.

Instead, there are lots of foreseeable task items related to the materials science aspects of electrochemistry. The list to be given below can be derived both from a statistics of the

László Péter

peter.laszlo@wigner.hu

1 Wigner Research Centre for Physics, Konkoly-Thege út 29-33, Budapest H-1121, Hungary electrochemistry-related publications and social needs, the latter being equally related to industrial interests and environmental concerns. Being aware of the fact that such lists are always arbitrary, it hopefully offers a wide enough topical selection. Another reason for putting materials science to the forefront of the electrochemical research is the change of scientific funding in the twenty-first century. Methodologyoriented funding opportunities became more and more scarce, and the open calls tend to require a high social benefit. The latter can be fulfilled with production-motivated research where the benefit is obvious and can be realized within a short time after solving a problem, should the road to the solution require decades.

In the opinion of the author, the following topics will attract much attention in the near future.

\section{Intercalation-based electrode materials with sodium ion uptake/release for rechargeable batteries}

The limited availability of the lithium on earth may soon become a bottleneck of the further spread of light rechargeable batteries. Although the energy density performance of the sodium ion batteries is weaker than that of the lithium batteries merely because of the difference in their molar weight, the virtually unlimited availability of sodium salts may compensate this disadvantage. Since not only the weight but the required excess volume of the sodium ion is also larger than that of lithium when intercalation takes place, the mechanical disintegration is a prominent problem for sodium-intercalating bulk electrode materials. This drawback can be compensated by producing highly dispersed host materials that tolerate 
volume expansion/contraction better than their bulk form. This anticipates that nano-engineering will play an important role in this development in line with electrochemistry.

\section{Improvement of photoelectrochemical energy conversion efficiency}

Application of renewable energy resources is a key question because of the limited availability of non-renewable ones, nature conservation, and environmental sustainability. Photoelectrochemical devices must be significantly improved, including both photovoltaic devices for direct energy conversion and photoelectrocatalytic systems for, e.g., hydrogen production and carbon dioxide reduction.

\section{Development of widely available, durable, and inexpensive electrocatalysts for fuel cells}

One of the limiting factors in the operation of several types of fuel cells is the sluggish nature of the oxygen reduction and the sensitivity of the noble metal-containing catalysts for poisoning materials. There is an immense demand for the replacement of the noble metal catalysts for less expensive and even more efficient catalysts. The research in this field deals with a wide range of materials, ranging from metal alloys through polymers to various composites. Since the catalyst particles have to be embedded into a complex system where transport of various components is involved, catalysts should be available in a highly dispersed form, which emphasizes the nanotechnological aspects of this field.

\section{Replacement of undesired or banned galvanic coating methods}

Metal coatings obtained by electroplating seem to be indispensable in both everyday life and high-tech industries. However, environmental issues drive lawmakers to gradually ban various materials. This list is quite significant, including cadmium-containing coating in general, baths based on $\mathrm{Cr}(\mathrm{VI})$ species, and the row is likely to be continued with $\mathrm{Ni}$ if we take into account the large percent of the population who produce allergic symptoms for Ni. Although the military and aerospace sector remained exempt from such regulation, there is a huge market demand for, e.g., easily operable plating systems containing $\mathrm{Cr}$ (III) species without expensive solvents (like ionic liquids). Besides, other systems with composite ("smart") coatings for corrosion protection should also be dealt with.

\section{Fast-charging technologies}

Due to the popularization of the portable electronic devices and the blow-up of the range of their functionality list, their energy consumption increases. In parallel, these devices became nearly an extension of human that are not dispensable even for short periods. Therefore, the charging rate of their batteries is a key issue, and so is for the field of electromobility. The development of safe fast-charging methods requires an involved cooperation of electrical engineers and electrochemists where the understanding of the electrochemical phenomena is a key issue.

\section{Education}

Although the sectioned list could be finished here, there is one further important issue that should not be neglected. This is the education of electrochemistry. The reason why it is particularly important is that the proliferation of new scientific and technological information that require time in education narrows the opportunity for didactic education of traditional fields. Classical electrochemistry is unfortunately one candidate for such sacrifice, although it is obvious that neither of the fields listed above can be studied in detail without an electrochemistry knowledge of solid ground. The complex nature of the fields listed also means that electrochemistry alone is not sufficient to cope with all problems - however, there cannot be a significant improvement in either of them without electrochemistry. Scientists having a good electrochemistry background have to adopt the mission of being the flag carriers of not only the research but of the electrochemistry education, too.

Funding Information Open access funding provided by Wigner Research Centre for Physics.

Open Access This article is licensed under a Creative Commons Attribution 4.0 International License, which permits use, sharing, adaptation, distribution and reproduction in any medium or format, as long as you give appropriate credit to the original author(s) and the source, provide a link to the Creative Commons licence, and indicate if changes were made. The images or other third party material in this article are included in the article's Creative Commons licence, unless indicated otherwise in a credit line to the material. If material is not included in the article's Creative Commons licence and your intended use is not permitted by statutory regulation or exceeds the permitted use, you will need to obtain permission directly from the copyright holder. To view a copy of this licence, visit http://creativecommons.org/licenses/by/4.0/. 